Tania Mercedes Ortiz-Valencia; Verónica Paulina Moreno-Narváez; Jaime Fabián Díaz-Córdova

DOI $10.35381 / \mathrm{cm} . v 6 \mathrm{i} 2.377$

\title{
Reconocimiento y valoración de activos biológicos en el sector ganadero
} aplicando costos ABC

\section{Recognition and valuation of biological assets in the livestock sector applying ABC costs}

\author{
Tania Mercedes Ortiz-Valencia \\ tania.ortiz87@est.ucacue.edu.ec \\ Universidad Católica de Cuenca, Cuenca \\ Ecuador \\ https://orcid.org/0000-0002-3288-9508 \\ Verónica Paulina Moreno-Narváez \\ veronica.moreno@ucacue.edu.ec \\ Universidad Católica de Cuenca, Cuenca \\ Ecuador \\ https://orcid.org/0000-0003-1517-6124 \\ Jaime Fabián Díaz-Córdova \\ jfdiazc@psg.ucacue.edu.ec \\ Universidad Católica de Cuenca, Cuenca \\ Ecuador \\ https://orcid.org/0000-0002-5406-4160
}

Recibido: 05 de agosto de 2020

Aprobado: 01 de octubre de 2020 


\title{
RESUMEN
}

El objetivo de este estudio es proponer un sistema de reconocimiento y valoración de activos biológico para el sector ganadero de la provincia del Azuay basado en costos $A B C$, cuyo resultado permite identificar los costos históricos del ganado por actividades y comparar con lo determinado por las normas contables internaciones como las NIIF donde se observa resultados positivos al comparar con el valor razonable con la contabilización. De tipo descriptiva no experimental. Los costos $A B C$ es la mejor técnica de manejo de recursos, pues permite conocer el desarrollo económico de la empresa e identificar los factores que afectan a los costos de producción, entre los más relevantes, la materia primera, el talento humano o los costos indirectos. Además, reconoce las actividades importantes, para saber aprovecharlas, buscar innovación o valor agregado a las mismas.

Descriptores: Toma de decisiones; normativa; ganado vacuno; sistema; producción (Palabras de Tesauro UNESCO).

\begin{abstract}
The objective of this study is to propose a system for the recognition and valuation of biological assets for the livestock sector of the province of Azuay based on ABC costs, the result of which allows identifying the historical costs of livestock by activities and comparing with those determined by accounting standards. Internationals such as IFRS where positive results are observed when comparing the fair value with the accounting. Non-experimental descriptive type. ABC costs is the best resource management technique, since it allows to know the economic development of the company and identify the factors that affect production costs, among the most relevant, the raw material, human talent or indirect costs. In addition, it recognizes the important activities, to know how to take advantage of them, seek innovation or added value to them.
\end{abstract}

Descriptors: Decision making; normative; cattle; system; production (Words from UNESCO Thesaurus). 
Tania Mercedes Ortiz-Valencia; Verónica Paulina Moreno-Narváez; Jaime Fabián Díaz-Córdova

\section{INTRODUCCIÓN}

La crisis económica palpada en el Ecuador a partir del año 2000 con la dolarización, ha perjudicado al sector agrícola, pues a partir de la fecha, los precios de los productos se incrementaron de forma notable, además, el calentamiento global o la contaminación ha sido uno de los factores trascendentes para que las personas dejen de consumir productos agrícolas, pues se sabe que, por este evento importante los alimentos son madurados con químicos para acelerar su crecimiento y su venta. Sin embargo, a pesar de la recesión de la economía, el país sigue siendo exportador de flores, petróleo, camarón, banano, cacao. En tal sentido, se cuenta con varios productos agrícolas que son de gran rentabilidad para las empresas, se espera que los pequeños y grandes comerciantes apunten a las exportaciones a costos bien definidos que generen ingresos y rentabilidad. Asimismo, el Ministerio de Agricultura, Ganadería y Pesca MAGAP, cuenta con programas y servicios para el apoyo de la agricultura, valoración del trabajo en el campo y generación de fuentes de ingresos.

Hoy en día, la falta de visualización de la agricultura como una ciencia de estudio, la desactualización tecnológica y la carencia de un sistema de costeo, ha impedido que los agricultores produzcan sus sembríos o animales con valores reales, por tanto, sus ventas se fijan en referencia a los precios del mercado, además, el calentamiento global es uno de los factores serios y de alto riesgo para la agricultura pues por este evento los insumos agrícolas elevan el costo del animal o planta viva.

Por otra parte, la contratación de la mano de obra barata, llamada así, a las personas que trabajan sin los beneficios de ley, es un factor perjudicial al momento de definir el costo del ganado. Ante esta situación que perjudica el buen desarrollo de un sistema de costeo agrícola, es importante analizar si ¿se está utilizando de forma efectiva la mano de obra?, ¿se capacita al personal a fin de que se maneje de forma eficiente los recursos agrícolas que se adquieren?, ¿cómo se logra mejorar la asignación de costos para una buena toma de decisiones?, ¿a qué segmento de mercado se direcciona los productos? 
Tania Mercedes Ortiz-Valencia; Verónica Paulina Moreno-Narváez; Jaime Fabián Díaz-Córdova

Es claro que los sucesos que impiden el desarrollo de la agricultura varían en el tiempo, ante la situación económica del país o las directrices gubernamentales que existan, sin embargo, la buena administración interna maneja un sistema de costos que permita la optimización de los recursos monetarios, el desarrollo de la competitividad y sostenibilidad de las empresas agrícolas o de los pequeños productores del país. Lo importante como bien lo menciona (Garzón, 2015), es conocer si los ganaderos ganan o pierden en la crianza de sus animales, a pesar de no contar con métodos técnicos que oriente el desarrollo de su negocio.

Bajo este contexto, el objetivo del presente artículo es diseñar un sistema de costeo basado en actividades en el marco de las Normas Internacionales de Información Financiera NIIF para el sector ganadero de la provincia del Azuay como mecanismo efectivo en la toma de decisiones.

\section{Referencial teórico}

\section{Modelo de costeo ABC y su aplicación}

Los sistemas de costos en la antigüedad, fueron diseñados por ingenieros que buscaban obtener los valores reales de los productos que ofrecían, sin embargo sus primeras practicas se empezaban a dificultar cuando se pretendía abrir nuevas líneas de productos, no obstante, en el año 1950, existieron investigaciones que comprobaron que los costos son trascendentales para una acertada toma de decisiones (Galarza, Narváez, \& Erazo, 2019).

Según el autor (Del Río, 1996) menciona a la palabra costos desde dos perspectivas, la primera la define como la suma de los esfuerzos económicos o físicos que se invierten para obtener algo; y la segunda visión indica que son los recursos que se sacrifican para obtener beneficios económicos adicionales de lo desembolsado. No obstante, los costos para (Ramírez, 2008) son las erogaciones que realiza una persona para obtener beneficios futuros siempre que mantengan una planeación, control y toma de decisiones. 
Tania Mercedes Ortiz-Valencia; Verónica Paulina Moreno-Narváez; Jaime Fabián Díaz-Córdova

Sin desmerecer la opinión de los autores, los costos no siempre conllevan a obtener beneficios económicos, pues al no realizar un análisis de inversiones y una buena toma de decisiones, estos costos llegarían a ser una perdida para las empresas.

En otro sentido, la ganadería busca mejorar cada uno de los procesos mediante la reducción de sus costos y la optimización de sus recursos, por tanto, como menciona (Bustamante, 2015) el reconocimiento de cada uno de los factores que originan costos en cada departamento permite asignar de forma efectiva, los valores a los productos y servicios. Atendiendo a esta consideración, para desarrollar de mejor manera los costos del área ganadera, se señala al sistema de costos basado en actividades o Costeo ABC, como el sistema apropiado para el manejo del ganado, además haciendo alusión a lo que indica el autor los costos $A B C$ permiten costear las actividades y procesos con base a los recursos que se utilizan, para con ello, asignar el costo a los productos o servicios que se ofertan.

Este sistema de costeo facilita a los empresarios, a conocer el movimiento de cada una de sus actividades y procesos; identificar si alguna de ellas no entrega ningún valor, para ser excluida; buscar nuevas estrategias y mejorar las que ya se encuentran establecidas; analizar los recursos que se requieren en cada centro de costos; $y$, sin duda, permite el análisis y la toma de decisiones para mejorar la rentabilidad de los negocios.

Ahora bien, el costeo $A B C$, requiere de actividades, es decir de un conjunto de tareas que se les asigne a los trabajadores para obtener el producto; cada actividad tiene objeto de costo, que permite optimizar los recursos y tomar decisiones de ser necesarias. En tal sentido, se debe tener presente los elementos del costo, tales como: materia prima, mano de obra y costos indirectos de fabricación (CIF), y reconocer la diferencia con el gasto, la falta de conocimiento de cada uno de los elementos, ocasiona pérdidas económicas, cese de funciones o liquidaciones de las empresas (Mogrovejo, Narváez , \& Erazo , 2019). Materia prima: según el criterio del mismo autor, la materia prima se la reconoce por ser un elemento físico y medible, por lo tanto, el valor monetario que se pague por su 
Tania Mercedes Ortiz-Valencia; Verónica Paulina Moreno-Narváez; Jaime Fabián Díaz-Córdova

adquisición, será considerado dentro del costo de un producto de forma directa, para el ámbito ganadero, se podría considerar materia prima al forraje, medicina, insumos para la salud animal, balanceado, pajuelas para inseminaciones artificiales, pastos, entre otros.

Mano de obra: es el esfuerzo físico realizado por las personas para la obtención de un producto o servicio. En ganadería, la mano de obra es considerada al ordeñador o el personal de pastoreo. Además, considerando la opinión de (Arrelano, Quispe, Ayaviri, \& Escobar, 2017), el personal de contabilidad junto con los técnicos responsables del hato ganadero, deben asignar y clasificar qué parte de este esfuerzo corresponde a costos de producción, administración o ventas. Del mismo modo y partiendo del criterio de los autores, se debe tener claro que, la mano de obra directa se considera el personal que se encuentran vinculados en la producción, mientras que la mano de obra indirecta son las personas que tienen un costo significativo en el producto y no participan en la transformación del mismo.

Costos indirectos de fabricación: corresponden a todos los costos que no son cuantificables de forma clara pero intervienen en la producción y en la transformación de un producto. Los costos indirectos cuentan con tres conceptos fundamentales, el primero es el material indirecto, que no es más que los insumos necesarios para la producción con un valor poco representativo, el segundo es la mano de obra indirecta y por último, los consumos de fabricación, que son los erogaciones por consumos de servicios públicos, arriendos, depreciaciones, entre otros (Arias, Portilla, \& Fernández, 2010). Una vez identificado los elementos del costo, se requiere conocer al costo fijo. Según (Lalangui, Eras, \& Burgos, 2017) son valores estáticos y no dependen de la actividad de la empresa ni del volumen de la producción, cuando los costos fijos son altos, pueden alterar en gran medida al valor del ganado, por lo tanto, impulsa al empresario a incrementar el volumen de sus ingresos, mediante el sistema $A B C$, los costos fijos son añadidos al proceso productivo. 
Tania Mercedes Ortiz-Valencia; Verónica Paulina Moreno-Narváez; Jaime Fabián Díaz-Córdova

Los costos drivers: también conocidos como inductores es una unidad de medición, permiten conducir un costo a una actividad determinada para ello se debe conocer la naturaleza del costo antes de su asignación, además los inductores deber ser confiables y permanecer en el tiempo a fin de poder comparar valores entre periodos, los más relevantes que se considera para el área de la agricultura es el número de horas máquina, horas hombre, metros de extensión de terreno, entre otros (Delicio, 1998).

Una vez reconocido las variables para el costeo $A B C$, las empresas deben estructurar los pasos a seguir para costear de forma clara y verás todos los recursos económicos, por lo tanto se debe considerar una línea de dirección de reconocer las actividades y objetos de costo, asignar los costos a cada centro de costos, determinar el costo de producción total, manejar un programa de comercialización, evaluar el sistema y tomar decisiones.

\section{Reconocimiento y medición de los activos biológicos con base a la normativa}

La Junta de Normas Internacionales de Contabilidad, crea al ámbito agrícola bajo la adopción de las NIC con el numeral 41, se estable en el año 2000 y se incorpora de forma obligatoria en el Ecuador en el año 2006 bajo el control de la Superintendencia de compañías (Quizhpi, Nárvaez, \& Erazo, 2019). La normativa permite conocer y diferenciar algunos términos significativos, entre ellos costo de venta, actividad agrícola, activos biológicos, etcétera.

Según los criterios de (Hernández, Núñez, \& Zapata, 2017), las empresas agrícolas manejan sus finanzas mediante costos históricos o a valor razonable, sin embargo, la ejecución de análisis a partir de manejos históricos, no permite reconocer con certeza los resultados económicos en tiempo real, ya que sus procesos productivos y las situaciones ambientales son cambiantes en cada periodo fiscal. Por tal razón, se sugiere el manejo económico a valor razonable, pues con este enfoque, existen algunas ventajas entre ellas, una mejor valoración de los activos biológicos, oportunidad de apertura de 
Tania Mercedes Ortiz-Valencia; Verónica Paulina Moreno-Narváez; Jaime Fabián Díaz-Córdova

mercados internacionales y la posibilidad de negociación en mercados activos del país. No obstante, varias empresas tienen limitaciones para costear su ganado, entre ellas, la falta de un sistema contable analítico, desarrollo de la información contable sin la consideración de la NIC 41, escases de políticas contables bien definidas, entre otros. Sobre la base de las ideas expuestas, las Normas Internacionales de Contabilidad (NIC), mencionan a la actividad agrícola como, la gestión necesaria para el desarrollo de animales o plantas vivas, como activos biológicos dirigidos para la producción o comercialización. Al respecto, (García, López, \& Ceron, 2015) mencionan que existe un ciclo evolutivo rígido, el mismo que comprende los procesos de crecimiento, producción y reproducción, estos afectan a la transformación biológica de manera cualitativa y cuantitativa ya sea de forma positiva o negativa en los costos de producción. Ante estas consideraciones, (KPMG Auditores Consultores Ltda, 2008) menciona que los activos biológicos son plantas y animales vivos capaces de experimentar transformaciones biológicas y brindar productos agrícolas que se contabilizan como existencias. Según (Caba \& Caba, 2008), la NIC 41 identifica al activo biológico de extenso periodo de crecimiento, degradación, producción y procreación, además, la norma reconoce a un activo biológico si cumple tres requisitos básicos el primero que sean propiedad de la empresa y que provengan de ejercicios pasados; los activos deben contar con registros formales de propiedad, el segundo que generen un beneficio económico a futuro y el tercero la facilidad de valoración de forma fiable o a valor razonable.

Características de los activos biológicos: tomando en consideración el criterio de (Jiménez, 2017) es importante identificar y clasificar los cambios del ganado, mientras el animal ofrezca producción o reproducción, se le considera activo biológico, sin embargo, al llegar a la edad aproximada de 60 meses y su finalidad no sea un beneficio económico, se convierte en propiedad, plata y equipo (NIC 16), lo ideal es identificar que el animal no se convierta en gastos improductivos. 
Tania Mercedes Ortiz-Valencia; Verónica Paulina Moreno-Narváez; Jaime Fabián Díaz-Córdova

Medición: el (Comité de Normas Internacionales de Contabilidad (IASCF), 2001) señala que el activo biológico debe ser medido en su reconocimiento inicial, sea por venta o nacimientos, y en su reconocimiento final a valor razonable menos los costos de venta. El valor razonable, es un valor o importe que un bien cotiza en un mercado activo, en este contexto, como parte de la información a revelar, las ganancias o pérdidas del ganado calculado a valor razonable deben incluirse y evidenciarse en los informes económicos del periodo así como una descripción detallada del ganado dependiendo del grupo al que pertenezca.

Uno de los problemas que se enfrenta en la actualidad al momento de costear el ganado o sus productos derivados, es que las entidades gubernamentales o asociaciones fijan precios referenciales para expender los activos biológicos, no obstante, la calidad ofertada de ganado varia de una empresa a otra, estos valores fijados permiten en algunas ocasiones cubrir costos y no obtener ninguna ganancia, ante esta situación, los propietarios se ven obligados a disminuir la calidad de sus productos con el fin de obtener beneficios económicos.

\section{Caracterización de los sistemas de producción del ganado vacuno}

Como mencionan (Pozo \& Sarmiento, 2012), la ganadería es el desarrollo científico y tecnológico que ha permitido aumentar la eficacia de la cría de ganado, mejorando las condiciones sanitarias y en general las condiciones de mantenimiento de los animales, por su parte, (Mafla, 2015) considera que la ganadería tiene vínculo directo con producción y colocación en el mercado de productos que son adquiridos para su faena o que son derivados de animales como los lácteos en especial la leche. Desde otro punto de vista la ganadería cubre diversas necesidades de consumo, que requieren de distintos tipos de segmentos de ganado como terneros, novillos, vacuno mayor, entre otros.

Considerando los aportes de (Peralta, Juscamaita, \& Meza, 2016), el ganado es una buena fuente de ingresos ya que produce carne, queso, yogurt, manjar y leche, así como 
Tania Mercedes Ortiz-Valencia; Verónica Paulina Moreno-Narváez; Jaime Fabián Díaz-Córdova

la elaboración de abonos biológicos mediante su excremento. Sin embargo, se debe vigilar los nutrientes que se brindan a los animales, a fin de disminuir riesgos ambientes y ahorrar costos que se requieran en la producción, además (Cruz, 2006) indica que el ganado vacuno se destaca por su gran potencial digestivo, que transforma los vegetales en nutrientes y minerales importantes para la salud del animal, en consecuencia, la buena crianza garantiza que vaconas se desarrolle con el propósito de ser fuente generadora de leche, y el animal macho tenga las condiciones necesarias para ser un buen semental. En otro sentido, (Morales, Morales, Andrade, \& Cevallos, 2020) propone mejorar la calidad productiva de su ganado con actualizaciones tecnológicas, es decir incorporando la aplicación de inteligencia de negocios M3S, este método facilita la toma de decisiones pues alerta a los productores él descarte de un animal por dificultades de reproducción, nivel bajo de producción de leche o enfermedades. Cuando un animal no gestiona ingresos económicos su permanencia dentro del hato ganadero comienza a ser un gasto improductivo.

El sector ganadero debe enfocarse en gestionar de forma adecuada algunas variables, como son: elevar el nivel de conocimientos técnicos y teóricos del personal a cargo de la ganadería, mientras no se capacite de forma periódica, los esfuerzos que se realicen en el área no obtendrá los resultados esperados. De igual forma el mantenerse actualizados permite a gestionar otro aspecto importante, la salud animal, pues la idea principal no es saber cómo resolver una enfermedad, sino más bien, saber prevenirla, por tanto, la alimentación del ganado es una de las condiciones necesarias para su buen desarrollo productivo, se debe manejar con sumo cuidado sus condiciones alimenticias, los horarios y manejo de pastoreo y ordeño, consumo de suplementos alimenticios y manejo sanitario. En el Ecuador, varias granjas de alto rendimiento lechero, manejan algunas razas importantes y reconocidas a nivel internacional: Holstein Friesian, Jersey, Brown Swiss y Montbeliar, estas razas se destacan por su alta producción de leche y su capacidad de fertilidad. En la provincia del Azuay, la mayoría de las haciendas manejan dos tipos de 
razas: el ganado Holstein, por cuanto posee un aspecto fisiológico destacado por sus manchas blancas, las mismas que permiten identificar la genética y el potencial del ganado, así como, el ganado Jersey que se reconoce por la apariencia de su cara corta, frente ancha y su pelaje es color café, ambas se adaptan al clima de la sierra sin perder su reconocimiento productivo (Jara \& Maldonado, 2011).

\section{MÉTODO}

En la investigación se adoptó un alcance descriptivo, el mismo que permitió detallar las variables de estudio y explicar de manera detallada el costeo, la normativa contable, la conformación ganadera, entre otras. Con base en las consideraciones de (Hernández, Fernández, \& Baptista, 2004), para obtener conocimientos generales de la agricultura y vincular con un análisis particular en el campo del ganado vacuno, el control de los costos a través del ámbito contable y enmarcar la información con la NIC 41 (Reyes, Narváez, Erazo, \& Giler, 2020).

La muestra estuvo conformada por 21 empresas ganaderas de la provincia del Azuay, con la participación de sus propietarios, administradores y contadores. Para el levantamiento de la información se elaboró una encuesta segmentada en las áreas: gerencia, contabilidad y ganadería. Se sometió a validación el cuestionario con el aporte de 5 expertos en el área de estudio, el mismo que contuvo preguntas con alternativas de respuesta de escala de Likert y dicotómicas, la confiabilidad del mismo se midió a través del coeficiente Alfa de Cronbach dando una fiabilidad de 0,90 en relación a los ítems consultados (Erazo \& Narváez, 2020). 
Tania Mercedes Ortiz-Valencia; Verónica Paulina Moreno-Narváez; Jaime Fabián Díaz-Córdova

\section{RESULTADOS}

Sistema de costeo: la mayor parte de empresas ganaderas de la provincia del Azuay manejan sus procesos productivos a partir de sistemas de costeo tales como órdenes de producción, basado en actividades y por procesos, sin embargo no todas definen de forma clara y confiable los costos de sus activos biológicos. Se observa que existe un nivel bajo de conocimientos del sistema de costeo $A B C$, por lo tanto, se evidencia la necesidad de una actualización de conocimientos al personal del área financiera (ver figura 1).

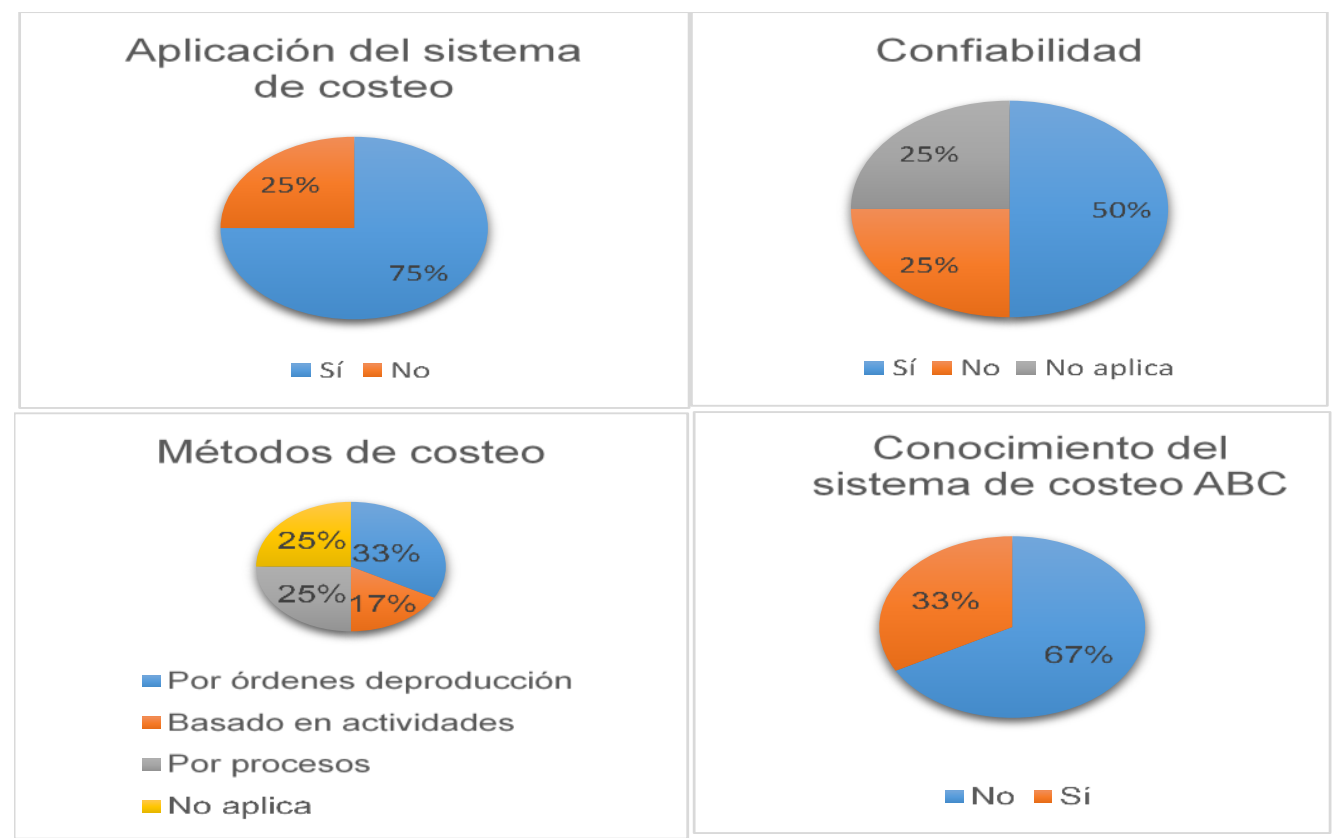

Figura 1. Análisis estadístico de aplicación de sistemas de costeo. 
Tania Mercedes Ortiz-Valencia; Verónica Paulina Moreno-Narváez; Jaime Fabián Díaz-Córdova

Normativa contable: la inobservancia de las NIIF por la mayoría de las empresas ganaderas del Azuay, no permiten obtener estados financieros comparables entre países distintos, no obstante del porcentaje de empresas que aplican la normativa realizan: la estimación, reconocimiento y medición de sus activos biológicos a partir de la NIC 41, manejan sus activos en base la NIC 2 de inventarios y aplican la NIIF 13, medición a valor razonable, en cuanto a los reportes que la NIC 41 solicita revelar, algunas empresas ejecutan las disposiciones de forma total y otras lo efectúan de forma parcial (ver figura 2).

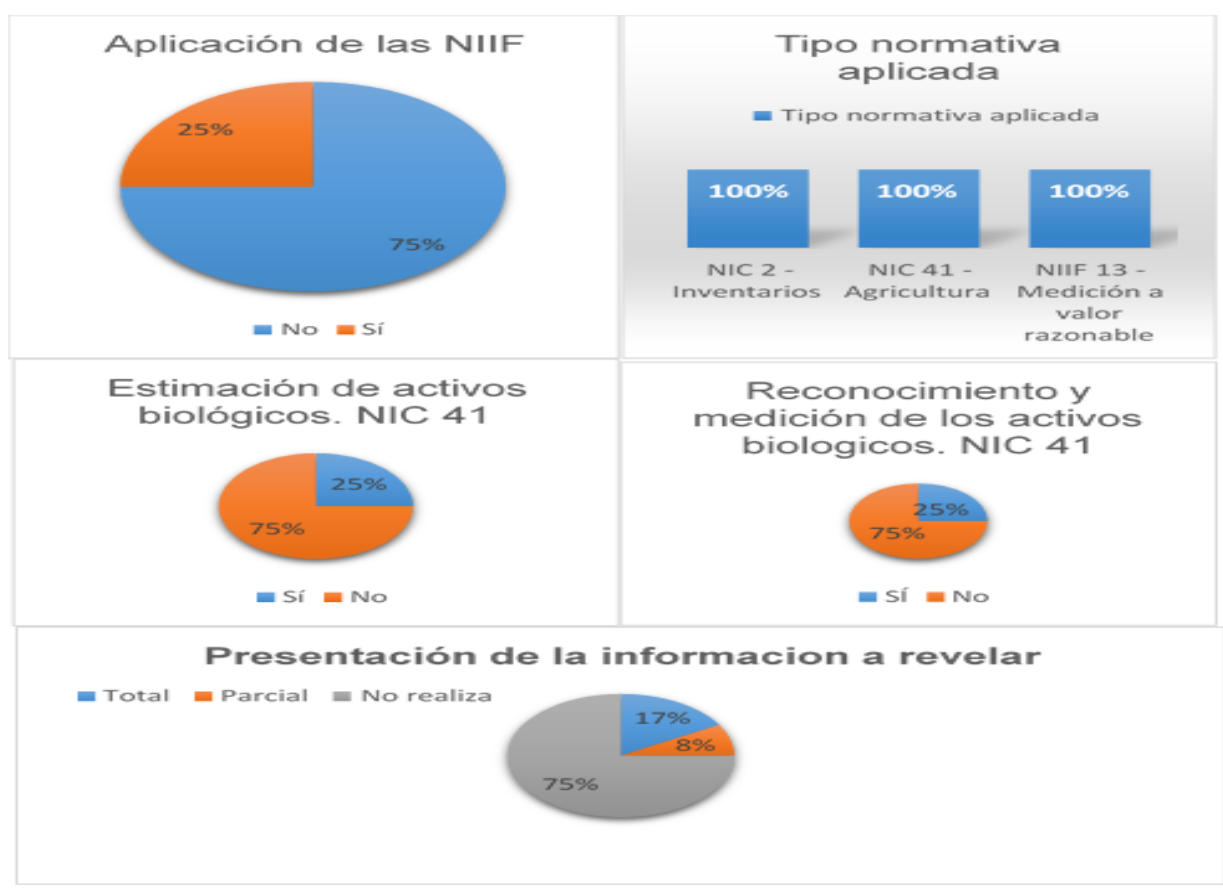

Figura 2. Análisis estadístico de aplicación de las NIIF. 
Tania Mercedes Ortiz-Valencia; Verónica Paulina Moreno-Narváez; Jaime Fabián Díaz-Córdova

Ganadería: el control y supervisión de los activos biológicos son responsabilidad del área contable como ganadera, por ello, se considera importante trabajar en equipo, en la figura 3 , se evidencia que la mayoría de empresas tienen concordancia en la información, el departamento de contabilidad tiene actualizado los inventarios, cantidad de hato ganadero, horas de trabajo, entre otros, y el área ganadera debe conocer el desarrollo productivo de los animales, con los datos de los centros de trabajo se debe comparar y obtener uniformidad.

En cuanto al manejo del talento humano, las funciones del personal están encaminadas a obtener resultados, se aprecia que un porcentaje alto no inspeccionan las labores de sus empleados, lo que dificulta el desarrollo económico y productivo del ganado. En porcentajes iguales se aprecia que las empresas realizan formación continua y otras no, si bien se busca conseguir efectos positivos en el trabajo de las personas, se necesita brindar programas de aprendizaje para el personal encargado del ganado vacuno en alimentación pecuaria, salud animal, reproducción genética, entre otros.

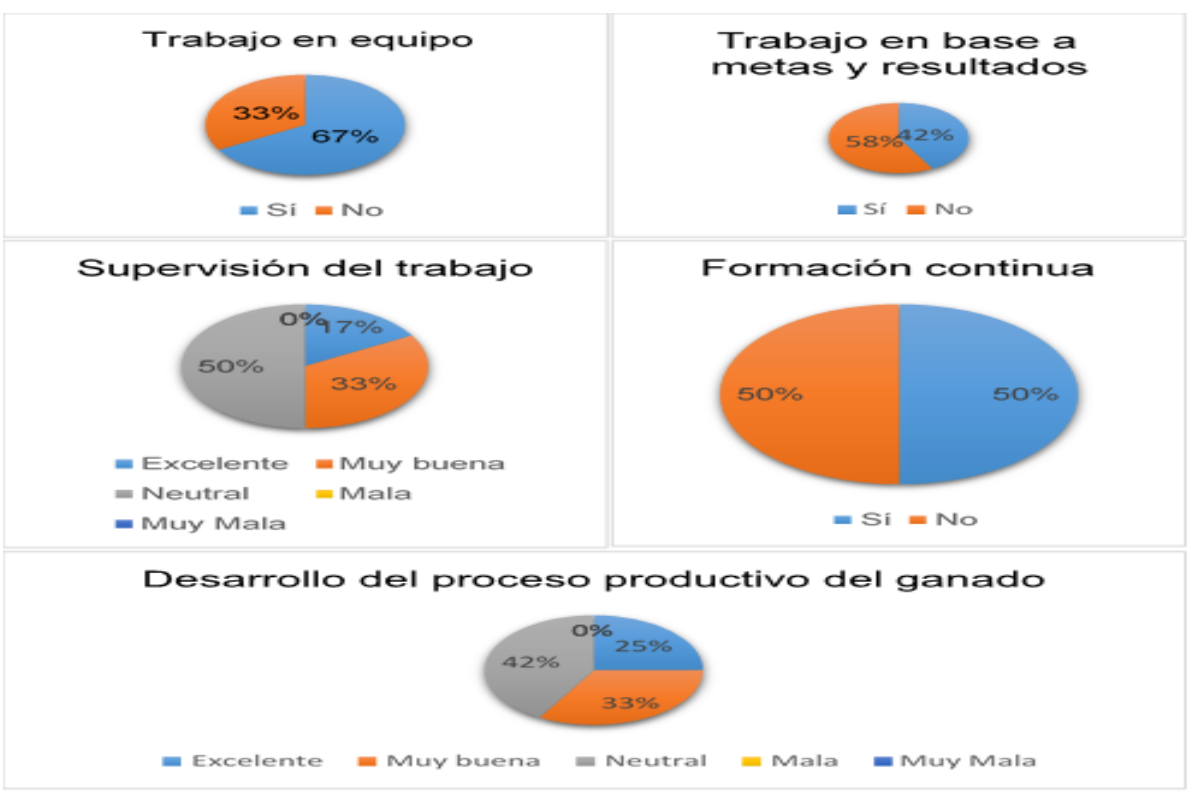

Figura 3. Análisis estadístico del desarrollo productivo de la ganadería. 
Gerencia: los gerentes o dueños de las sociedades ganaderas, buscan mejorar sus procesos productivos y tomar las mejores decisiones, se evidencia que el $75 \%$ de empresas obtienen información suficiente y necesaria con su sistema de costos y el $25 \%$ no. Desde la perspectiva de las ganancias que han obtenido por el desarrollo de su negocio, la mayoría se encuentran conformes o neutrales con la rentabilidad, sin embargo, esto podría mejorar si realiza los análisis financieros en tiempos oportunos, pues el $46 \%$ de compañías efectúan análisis mensuales, el no ejecutar evaluaciones tempranas se expone a un mal manejo económico y técnico que dificulte una acertada toma de decisiones.

\section{PROPUESTA}

A partir del análisis de los datos obtenidos, se propone un sistema de contabilidad basado en actividades para una empresa agropecuaria, se plantea la presentación de un diagrama de flujo para identificar los pasos a seguir del sistema de costeo, como se aprecia en la figura 4 se abarca información del proceso de compra, de producción, de ventas y la toma de decisiones, además considerando el apartado de la NIC 41, existen actividades que son analizadas a partir de esta normativa y se realiza una propuesta para la toma de decisiones por parte de la administración. 
CIENCIAMATRIA

Revista Interdisciplinaria de Humanidades, Educación, Ciencia y Tecnología

Año VI. Vol. VI. N². Edición Especial II. 2020

Hecho el depósito de ley: pp201602FA4721

ISSN-L: 2542-3029; ISSN: 2610-802X

Universidad Nacional Experimental Francisco de Miranda (UNEFM). Santa Ana de Coro. Venezuela

Tania Mercedes Ortiz-Valencia; Verónica Paulina Moreno-Narváez; Jaime Fabián Díaz-Córdova

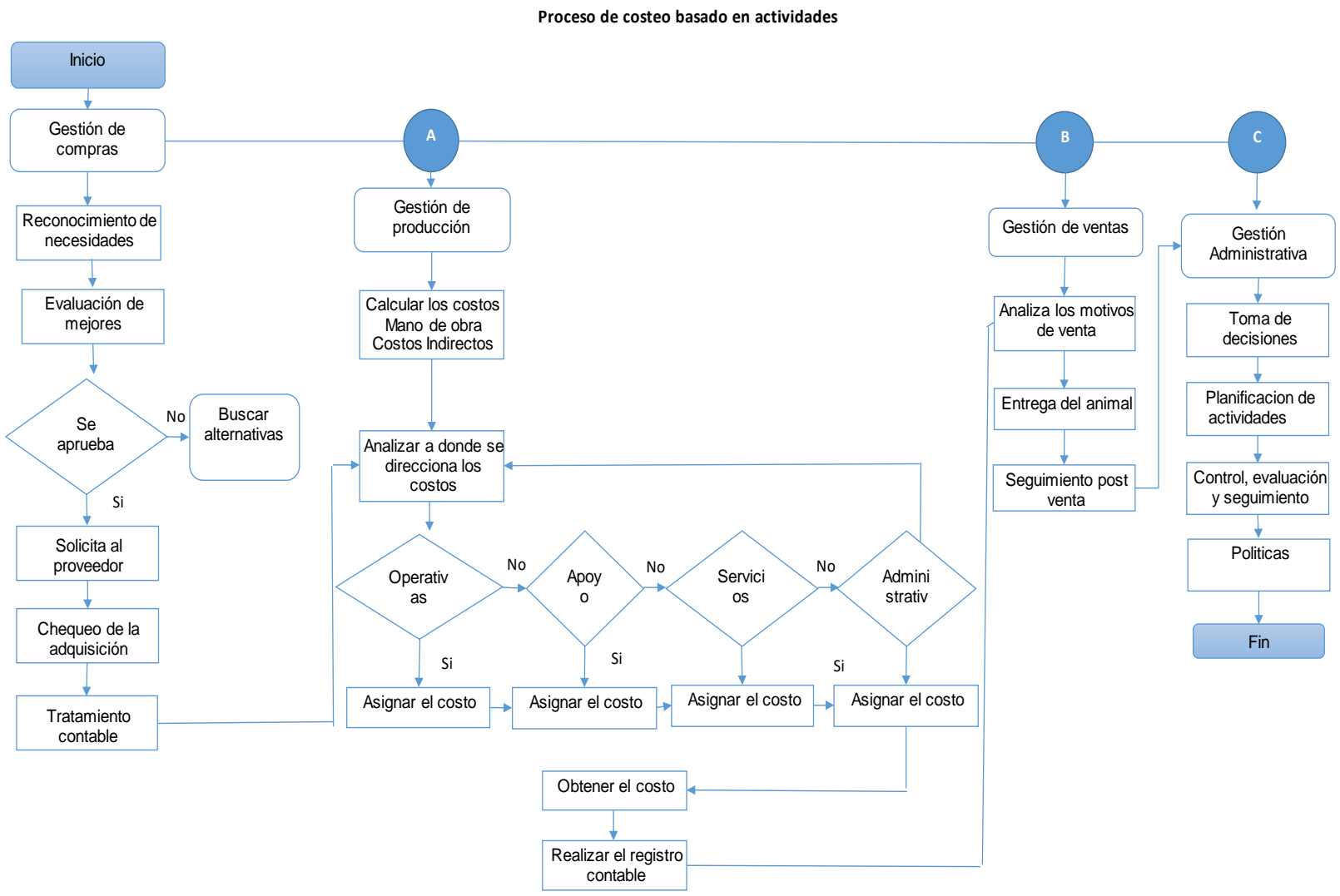

Figura 4. Diagrama de flujo del sistema de costos basado en actividades.

\section{Gestión de adquisición o compras}

En la figura 4 se propone iniciar un sistema de costeo desde la gestión de compras, aunque este proceso es muy amplio de analizar, las evaluaciones minuciosas de los insumos a adquirir resultan importantes para determinar desde el principio una buena genética, alimentación saludable y beneficios económicos futuros. En primera instancia, se debe realizar el reconocimiento de las necesidades, algunas de ellas son presupuestadas a tiempo, de forma seguida se debe buscar las mejores alternativas en el mercado, en calidad y costo, de existir la aprobación de la persona a cargo se solicita y chequea que el producto llegue en las condiciones pactadas. 
En el ámbito contable, según la NIC 41, cuando la empresa adquiere un semoviente, debe reconocer al animal como activo biológico, controlar como resultado de sucesos pasados, obtener futuros beneficios económicos y su valor razonable debe ser medido de forma confiable. Además, una vez adquirido el semoviente, se debe reducir los costos de adquisición. Para el caso de estudio, una empresa agropecuaria adquiere 5 becerros a 300 c/d, 5 vacas de engorde a $\$ 500,00$ y 5 vacas de producción a $\$ 1000$, para la transportación de los animales cobran 120,00 por animal. Con los datos proporcionados se realizan los cálculos necesarios para determinar el reconocimiento del activo biológico.

\section{Tabla 1.}

Asiento contable para reconocimiento inicial del activo biológico.

\begin{tabular}{ccrrr}
\hline Fecha & \multicolumn{1}{c}{ Detalle } & Auxiliar (\$) & Debe (\$) & Haber (\$) \\
\hline $\mathbf{0 1 / 0 5 / 2 0 2 0}$ & $\mathrm{X}$ & & $10.800,00$ & \\
& Activo biológico & $2.100,00$ & & \\
& Becerro & $3.100,00$ & \\
& Engorde & $5.600,00$ & \\
& Producción & & $10.800,00$ \\
& & & \\
& Pancos & & \\
& P/r adquisición de ganado incluido & & \\
& transporte &
\end{tabular}


CIENCIAMATRIA

Revista Interdisciplinaria de Humanidades, Educación, Ciencia y Tecnología

Año VI. Vol. VI. N². Edición Especial II. 2020

Hecho el depósito de ley: pp201602FA4721

ISSN-L: 2542-3029; ISSN: 2610-802X

Universidad Nacional Experimental Francisco de Miranda (UNEFM). Santa Ana de Coro. Venezuela

Tania Mercedes Ortiz-Valencia; Verónica Paulina Moreno-Narváez; Jaime Fabián Díaz-Córdova

\section{Gestión de producción}

En base al sistema de costeo basado en actividades, se debe realizar algunos pasos para la determinación del costo del producto, se observa en la figura 5, el proceso de sistema de costeo inicia desde la determinación y cálculo de la mano de obra, los costos indirectos de fabricación, el análisis de los centros de costo, reconocimiento de actividades, inductores, la asignación de costos y determinación de costos totales de producción.

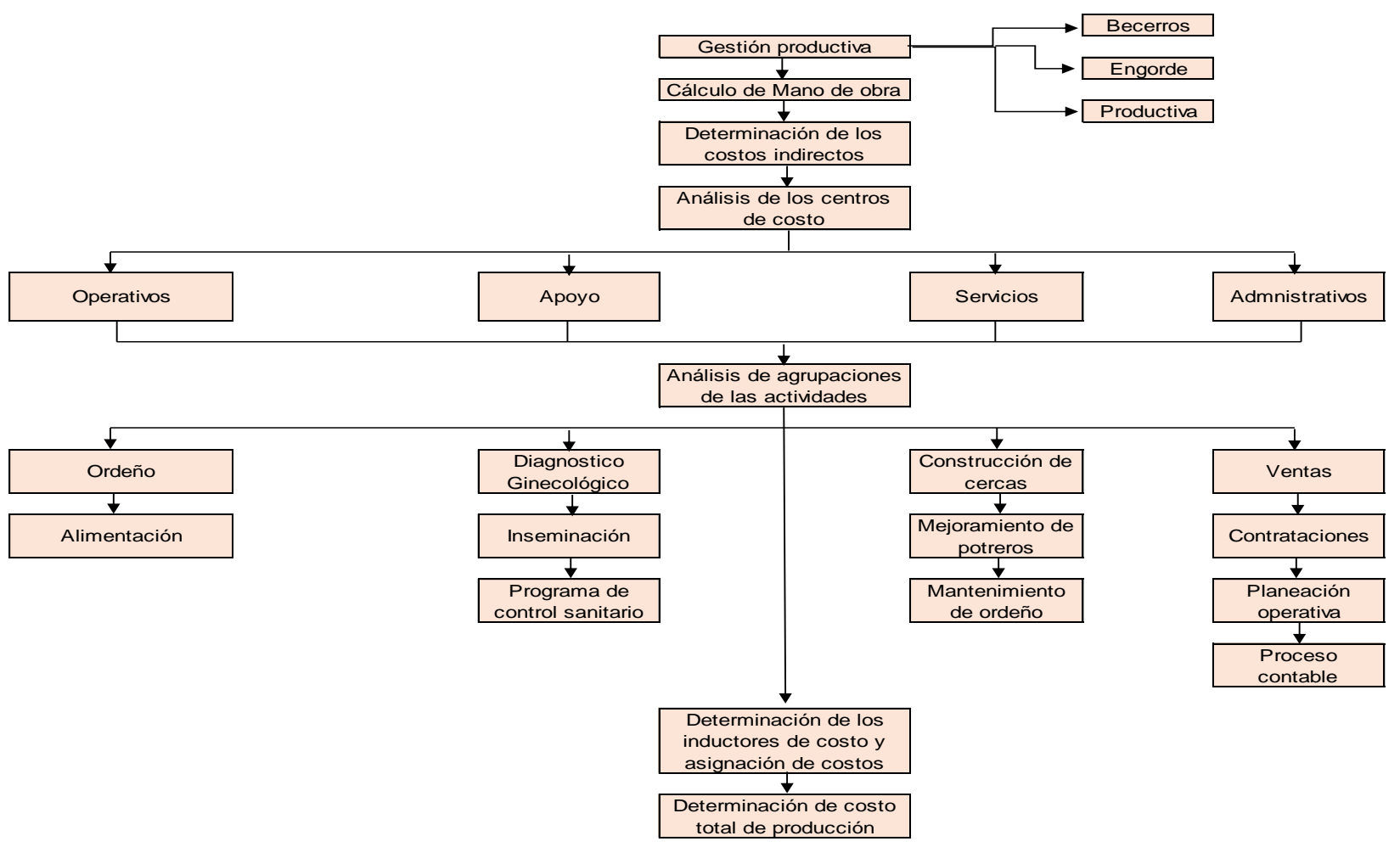

Figura 5. Sistema de costeo ABC. 
Tania Mercedes Ortiz-Valencia; Verónica Paulina Moreno-Narváez; Jaime Fabián Díaz-Córdova

\section{Cálculo de la mano de obra}

Para la investigación, se analiza un caso hipotético de estudio, se realiza el cálculo de la mano de obra individual debido a que el personal realiza varias actividades, por lo tanto, se requiere analizar las horas efectivas trabajadas en cada centro de costos operativos, apoyo y servicios e identificar si corresponde a costos directos o indirectos, además, se evalúa el número de horas de cada trabajador en cada centro de costos, se asigna el tiempo a partir de cifras porcentuales y se obtiene el costo de mano de obra.

\section{Tabla 2.}

Costo de mano de obra.

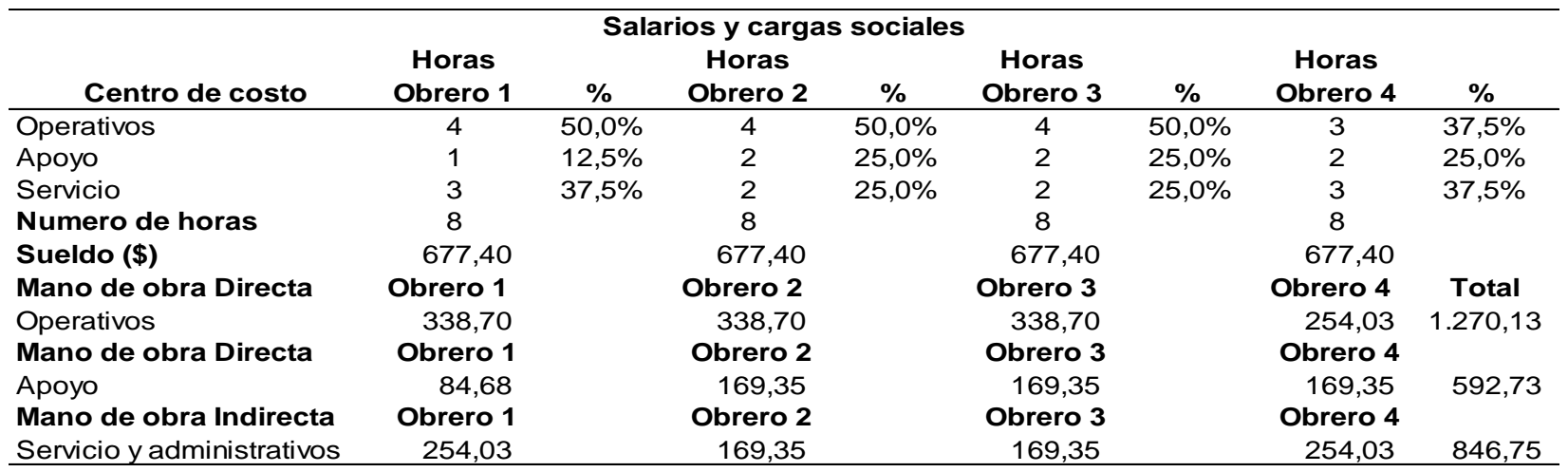

\section{Determinación de costos indirectos}

Tomando los datos de los estados financieros de la empresa agropecuaria, se obtiene los costos indirectos de un periodo de tiempo, con ello se identifica el centro de costos, las actividades y sus inductores para incorporar los rubros al costo total de producción, como se aprecia en la tabla 3, para la determinación de los costos por áreas, se debe estimar los costos en base al inductor definido y prorratear ha ganado becerros, engorde y productivos según los aspectos técnicos que estimen la empresa. 
CIENCIAMATRIA

Revista Interdisciplinaria de Humanidades, Educación, Ciencia y Tecnología

Año VI. Vol. VI. N². Edición Especial II. 2020

Hecho el depósito de ley: pp201602FA4721

ISSN-L: 2542-3029; ISSN: 2610-802X

Universidad Nacional Experimental Francisco de Miranda (UNEFM). Santa Ana de Coro. Venezuela

Tania Mercedes Ortiz-Valencia; Verónica Paulina Moreno-Narváez; Jaime Fabián Díaz-Córdova

Tabla 3.

Determinación de costos indirectos.

\begin{tabular}{|c|c|c|c|c|c|c|c|c|c|c|c|}
\hline Costos Indirectos & $\begin{array}{l}\text { Centro de } \\
\text { costos }\end{array}$ & Actividades & $\begin{array}{c}\text { Elementos } \\
\text { del costo }\end{array}$ & Inductor & Costo (\$) & $\begin{array}{l}\text { Administrativa } \\
\text { (\$) }\end{array}$ & $\begin{array}{c}\text { Comedor } \\
\text { (\$) }\end{array}$ & $\begin{array}{c}\text { Area } \\
\text { productiv } \\
\text { a } \\
(\$)\end{array}$ & $\begin{array}{c}\text { Area de } \\
\text { becerros } \\
(\$)\end{array}$ & $\begin{array}{c}\text { Area } \\
\text { engorde } \\
(\$)\end{array}$ & Total (\$) \\
\hline \multirow{2}{*}{$\begin{array}{l}\text { Mano de obra Indirecta } \\
\text { Insumos de limpieza }\end{array}$} & Servicios & $\begin{array}{c}\text { Mantenimient } \\
0\end{array}$ & $\mathrm{CIF}$ & \multirow{2}{*}{$\begin{array}{l}\text { Horas hombre } \\
\text { mtrs } 2\end{array}$} & \multirow{2}{*}{$\begin{array}{l}846,75 \\
500,00\end{array}$} & 635,06 & \multirow[b]{2}{*}{25,00} & 76,21 & \multirow{3}{*}{$\begin{array}{r}67,74 \\
125,00\end{array}$} & \multirow{3}{*}{$\begin{array}{r}67,74 \\
125,00\end{array}$} & 846,75 \\
\hline & Servicios & Mantenimient & $\mathrm{CIF}$ & & & 50,00 & & 175,00 & & & 500,00 \\
\hline Insumos de maternidac & Apoyo & $\begin{array}{l}\text { Programa de } \\
\text { reproducción }\end{array}$ & $\mathrm{CIF}$ & mtrs 2 & 160,00 & & & 160,00 & & & 160,00 \\
\hline Combustibles & Operativas & Alimentación & $\mathrm{CIF}$ & Horas maquina & 750,00 & & & 300,00 & 300,00 & 150,00 & 750,00 \\
\hline Energia eléctrica & Servicios & Mantenimient & $\mathrm{CIF}$ & mtrs 2 & 350,00 & 35,00 & 17,50 & 122,50 & 87,50 & 87,50 & 350,00 \\
\hline Agua & Servicios & Mantenimient & $\mathrm{CIF}$ & mtrs 2 & 525,00 & 52,50 & 26,25 & 183,75 & 131,25 & 131,25 & 525,00 \\
\hline \multirow[t]{2}{*}{ Maquinaria } & Servicios & ivlärller IIIIIIeril & $\mathrm{CIF}$ & Horas máquina & 540,00 & & & 216,00 & 216,00 & 108,00 & 540,00 \\
\hline & & & & Total & $3.671,75$ & 772,56 & 68,75 & $1.233,46$ & 927,49 & 669,49 & $3.671,75$ \\
\hline
\end{tabular}

\section{Centros de costo, actividades e inductores}

Para la determinación del centro de costo es necesario analizar las características organizativas de la producción, la empresa ganadera cuenta con centro operativo, de apoyo, de servicios y administrativos, cada uno de ellos tienen asignado actividades específicas. El centro de costos operativo, tiene actividades de ordeño de animales y alimentación; el de apoyo, programas de vacunación, reproducción y chequeos médicos, de servicios realizan todo el proceso de mantenimiento de espacios, equipos y construcciones, y administrativos encargados del análisis y supervisión de los demás centros. Como en todas las líneas de costeo, se debe establecer el inductor del costo o driver, es la base para la asignación de costos en la segmentación de ganado, para la empresa agropecuaria, como se aprecia en la tabla 4 se incorpora en cada centro de costos la mano de obra calculada (tabla 2) junto con los costos indirectos. 


\section{CIENCIAMATRIA}

Revista Interdisciplinaria de Humanidades, Educación, Ciencia y Tecnología

Año VI. Vol. VI. N². Edición Especial II. 2020

Hecho el depósito de ley: pp201602FA4721

ISSN-L: 2542-3029; ISSN: 2610-802X

Universidad Nacional Experimental Francisco de Miranda (UNEFM). Santa Ana de Coro. Venezuela

Tania Mercedes Ortiz-Valencia; Verónica Paulina Moreno-Narváez; Jaime Fabián Díaz-Córdova

Tabla 4.

Asignación de costos por actividades.

\begin{tabular}{|c|c|c|c|c|c|c|c|c|}
\hline Centro de costos & Actividades & $\begin{array}{l}\text { Elementos } \\
\text { del costo }\end{array}$ & Inductor & Costo (\$) & $\begin{array}{c}\text { Área } \\
\text { productiva (\$) }\end{array}$ & $\begin{array}{c}\text { Área de } \\
\text { levante } \\
(\$)\end{array}$ & $\begin{array}{c}\text { Área } \\
\text { engorde } \\
(\$) \\
\end{array}$ & Total (\$) \\
\hline \multirow{4}{*}{ Apoyo } & Chequeos médicos & MOD & $\begin{array}{l}\text { Número de } \\
\text { animales }\end{array}$ & 592,73 & 296,37 & 118,55 & 177,82 & 592,73 \\
\hline & Vacunación & MPD & $\begin{array}{l}\text { Número de } \\
\text { animales }\end{array}$ & 200,00 & 66,66 & 66,66 & 66,66 & 199,98 \\
\hline & & MPD & $\begin{array}{l}\text { Número de } \\
\text { animales }\end{array}$ & 100,00 & & & 100,00 & 100,00 \\
\hline & Reproducción & $\mathrm{CIF}$ & Mtrs 2 & 160,00 & & & $160,00^{\prime}$ & 160,00 \\
\hline \multirow{4}{*}{ Operativas } & Ordeño & MOD & $\begin{array}{l}\text { Número de } \\
\text { horas } \\
\text { mensuales } \\
\text { trabajadas } \\
\end{array}$ & $1.270,13$ & 423,33 & 317,53 & 529,26 & $1.270,13$ \\
\hline & & MPD & $\begin{array}{l}\mathrm{Kg} \text { de consumo } \\
\text { mensuales }\end{array}$ & 800,00 & 312,00 & 176,00 & 312,00 & 800,00 \\
\hline & Alimentación & MPD & $\begin{array}{c}\text { Metros } \\
\text { cuadrados de } \\
\text { consumo } \\
\text { mensuales }\end{array}$ & 250,00 & 50,00 & 100,00 & 100,00 & 250,00 \\
\hline & & $\mathrm{CIF}$ & Horas maquina & 750,00 & $300,00^{\circ}$ & 300,00 & 150,00 & 750,00 \\
\hline Servicios & Mantenimiento & $\mathrm{CIF}$ & $\begin{array}{c}\text { Metros } \\
\text { cuadrados de } \\
\text { consumo } \\
\text { mensuales } \\
\end{array}$ & $1.920,45$ & 773,46 & 627,49 & 519,50 & $1.920,45$ \\
\hline \multirow{3}{*}{ Administrativo } & & CIF & $\begin{array}{c}\text { Horas hombre } \\
\text { Metros }\end{array}$ & 635,06 & 211,67 & 211,73 & 211,67 & 635,06 \\
\hline & Administrativas & $\mathrm{CIF}$ & $\begin{array}{c}\text { cuadrados de } \\
\text { consumo } \\
\text { mensuales }\end{array}$ & 206,25 & 68,74 & 68,74 & 68,74 & 206,23 \\
\hline & & & Total & $6.884,62$ & $2.502,23$ & $1.986,70$ & $2.395,65$ & $6.884,58$ \\
\hline
\end{tabular}




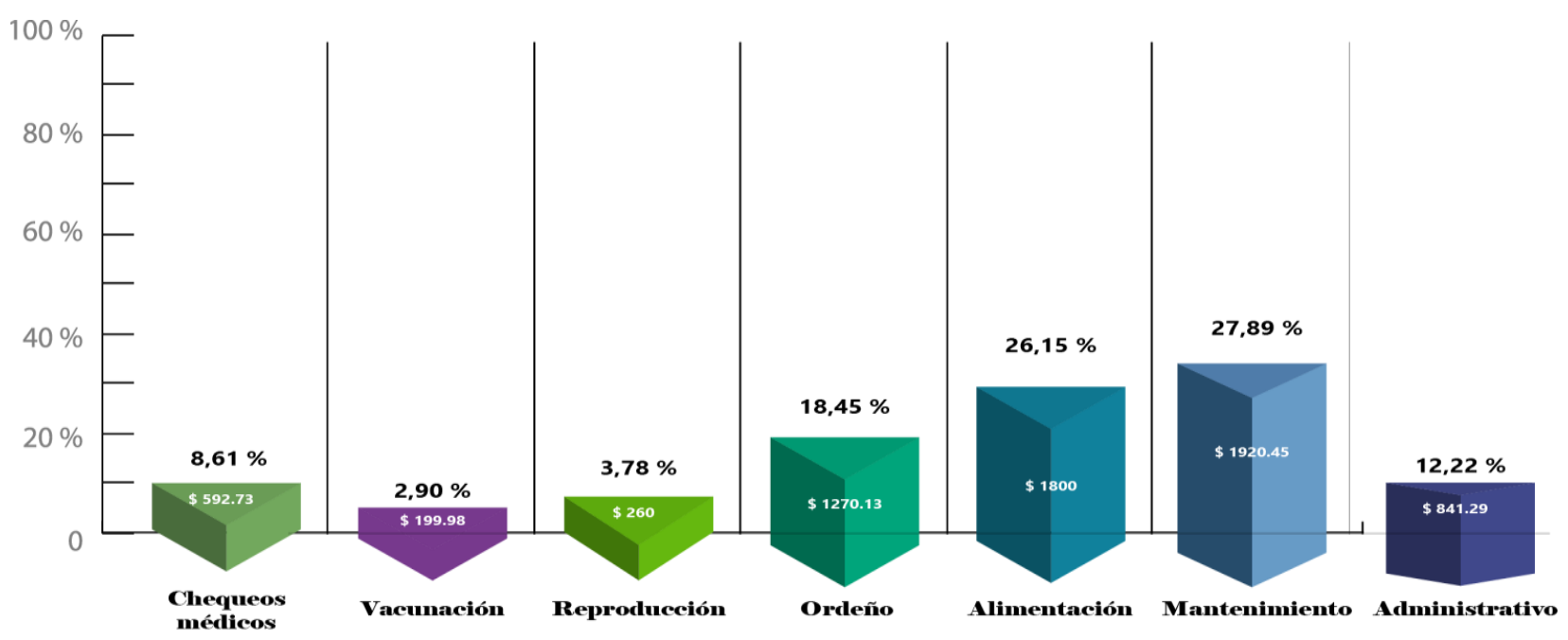

Figura 6. Representación gráfica del sistema de costeo ABC.

\section{Determinación del costo total}

Para la determinación de costo, se procede a calcular el costo total de producción, a partir de los centros de costos, segmentación de ganado y costos directos e indirectos, en la tabla 5 se aprecia los costos totales de becerros, engorde y productivos, con un hato ganadero 5 becerros, el costo de cada animal asciende a $\$ 397.43$; ganado de engorde en 5 cabezas de ganado su costo es de $\$ 532.61$ y las 5 vacas en reproducción su valor asciende a $\$ 447.45$. Cabe mencionar que los rubros de gastos administrativos, se incluyen en los costos de producción indirectos y con una valoración porcentual dividida en las tres segmentaciones de ganado. 
CIENCIAMATRIA

Revista Interdisciplinaria de Humanidades, Educación, Ciencia y Tecnología

Año VI. Vol. VI. N². Edición Especial II. 2020

Hecho el depósito de ley: pp201602FA4721

ISSN-L: 2542-3029; ISSN: 2610-802X

Universidad Nacional Experimental Francisco de Miranda (UNEFM). Santa Ana de Coro. Venezuela

Tania Mercedes Ortiz-Valencia; Verónica Paulina Moreno-Narváez; Jaime Fabián Díaz-Córdova

\section{Tabla 5 .}

Costo total de producción.

\begin{tabular}{|c|c|c|c|c|c|c|}
\hline \multirow[b]{2}{*}{ Centro de costos } & \multicolumn{3}{|c|}{ Costos Directos } & \multicolumn{3}{|c|}{ Costos Indirectos } \\
\hline & Becerros (\$) & roductivos $(\$$ & Engorde (\$) & Becerros (\$) & $\begin{array}{l}\text { Productivos } \\
\text { (\$) }\end{array}$ & Engorde (\$) \\
\hline Servicio & - & - & - & 627,49 & 773,46 & 519,49 \\
\hline Apoyo & 185,74 & 363,86 & 346,11 & - & 160,00 & - \\
\hline Operativas & 593,53 & 785,33 & 941,26 & 300,00 & 300,00 & 150,00 \\
\hline Administrativos & & & & 280,41 & 280,41 & 280,41 \\
\hline Total & 779,27 & $1.149,19$ & $1.287,37$ & $1.207,90$ & $1.513,87$ & 949,90 \\
\hline Segmentación & $\begin{array}{l}\text { Costo total } \\
\text { (\$) }\end{array}$ & $\begin{array}{l}\text { Costo por } \\
\text { animal (\$) }\end{array}$ & & & & \\
\hline Becerros & $1.987,17$ & 397,43 & & & & \\
\hline Engorde & $2.663,06$ & 532,61 & & & & \\
\hline Reproductivo & $2.237,27$ & 447,45 & & & & \\
\hline Total & $6.887,50$ & & & & & \\
\hline
\end{tabular}

Los costos de producción deben ser contabilizados en un periodo determinado, los activos biológicos en producción como cuenta deudora, contra los costos de producción considerando la clasificación de materia prima, mano de obra y costos indirectos de fabricación. 


\section{Tabla 6.}

Asiento contable de costos de producción.

\section{Detalle}

$-x-$

Activo Biológico

Becerros

Engorde

producción
Auxiliar (\$) Debe (\$) Haber (\$)

6887.50
1987.17

2663.06

2237.27

6887.50

Costos de producción

Materia prima

1350.00

Mano de obra

1862.86

Costos indirectos de fabricación

3674.64

$\mathrm{P} / \mathrm{r}$ reconocimiento final de los activos biológicos

\section{Gestión de ventas y su Reconocimiento final}

Según la NIC 41, los activos biológicos deben ser calculados en su reconocimiento final, a su valor razonable menos el costo de venta, por tal razón, en cada segmentación de ganado debe analizarse los valores que se encuentran cotizados en el mercado y a estos deducir los costos de producción, para la empresa agropecuaria obtiene costos totales en ganado becerro de $\$ 397.43$, engorde $\$ 532.61$ y de reproducción $\$ 447.45$; su valor razonable unitario es de $\$ 1200$ becerros, $\$ 1600$ engorde y $\$ 2500$ reproducción, el resultado total es una ganancia al final del periodo de $\$ 19612.50$, es decir al momento de vender el activo al precio de mercado, se obtendría una ganancia por su comercialización, por la venta de 5 becerros se obtiene $\$ 4012.83$, de 5 vacas engorde $\$ 5336.94$ y de 5 vacas en producción $\$ 10262.73$. 


\section{Tabla 7.}

Reconocimiento final.

\begin{tabular}{lclc}
\hline Ganado & $\begin{array}{c}\text { Valor } \\
\text { razonable }\end{array}$ & $\begin{array}{c}\text { Costo de } \\
\text { producción }\end{array}$ & Valor final \\
\hline Becerros & 6000.00 & 1987.17 & 4012.83 \\
Engorde & 8000.00 & 2663.06 & 5336.94 \\
producción & 12500.00 & 2237.27 & 10262.73 \\
& & &
\end{tabular}

\section{Gestión administrativa}

Aunque a las empresas, es difícil detectar errores en sus actividades, efectuar los correctivos a tiempo permite obtener mayores beneficios, por tal motivo la empresa debe considerar algunos aspectos a identificar:

\section{Tabla 8 .}

Propuesta de toma de decisiones.

\begin{tabular}{ll}
\hline \multicolumn{1}{c}{ Aspectos } & \multicolumn{1}{c}{ Propuesta } \\
\hline Administrativos & Definir de forma clara un modelo de sistema de costos, \\
& siendo el costeo ABC, la mejor opción para las empresas \\
& ganaderas. \\
- & Invertir en software o actualizar el existente que contengan \\
& los modelos necesarios para obtener información. \\
$\bullet$ & Realizar control de inventarios \\
& Solicitar un análisis a tiempo, con el fin de prevenir riesgos \\
& de liquidez \\
& Comunicar el modelo de gestión de actividades, y las \\
& responsabilidades que se encuentran a su cargo.
\end{tabular}


Normativa

Comunicación

Ganadería
- Capacitación continua de temas referentes a su cargo para evitar pérdida de tiempo y recursos.

- Contar con manuales de funciones o control interno de cada actividad.

- Trabajar con los lineamientos de las Normas Internacionales de Contabilidad.

- Informar al personal acerca de los cambios que surjan en la economía de la empresa

- Considerar la opinión de los trabajadores y dar respuesta a sus sugerencias

- Mantener los estándares de calidad del hato ganadero

- Realizar programas de salud animal a fin de evitar enfermedad que perjudiquen a la economía del negocio

- Evitar la contaminación ambiental, este aspecto es vital para las empresas de perfil agrónomo.

- Evaluación pormenorizada de los animales, reconocer descartes y celos. 
Tania Mercedes Ortiz-Valencia; Verónica Paulina Moreno-Narváez; Jaime Fabián Díaz-Córdova

\section{DISCUSIÓN}

El sistema de costeo basado en actividades brindan la oportunidad de reconocer los costos del ganado no solo por sus elementos de costo sino también considera a los gastos administrativos, de venta y financieros, además identifica el costo que implica cada actividad con el fin de mejorar los procesos que representen un cuello de botella para la empresa.

Las Normas Internacionales de Información Financiera se han constituido en un vínculo de oportunidades de negocios a nivel nacional e internacional, además cuenta con un lenguaje confiable y comparable de los estados financieros. Conocer y aplicar los lineamientos y disposiciones de la NIC 41, mejora los costos de los activos biológicos desde su reconocimiento inicial hasta la venta.

En los campos ganaderos de la provincia del Azuay, se ha evidenciado la poca importancia hacia un buen manejo de costos, lo que ha conllevado a no definir de forma clara sus valores productivos, se apreció que las empresas no han incorporado la normativa internacional vigente, estos dos aspectos impiden que la gerencia obtenga información correcta de los costos productivos del ganado.

Es importante que exista uniformidad de los datos entre el área de producción y el área contable, además, es bueno evaluar el manejo del talento humano, aprovechar al máximo sus capacidades y brindarles la oportunidad de aprender de las innovaciones productivas que existen en el transcurso del tiempo. No obstante, el crecimiento económico y humano es responsabilidad compartida, por lo tanto, cada persona debe ejecutar su trabajo a conciencia y respetando las políticas institucionales.

La propuesta de manejo de costos con el sistema $A B C$ en la línea ganadera ha permitido evidenciar que los costos indirectos representan el 53\% en relación al costo total de producción, en él se incorpora los costos fijos que a deferencia del costeo tradicional no contemplan estos rubros por ello, este sistema permite definir con certeza el valor de un activo biológico. 
Tania Mercedes Ortiz-Valencia; Verónica Paulina Moreno-Narváez; Jaime Fabián Díaz-Córdova

Los costos $A B C$ es la mejor técnica de manejo de recursos, pues permite conocer el desarrollo económico de la empresa e identificar los factores que afectan a los costos de producción, entre los más relevantes, la materia primera, el talento humano o los costos indirectos. Además, reconoce las actividades importantes, para saber aprovecharlas, buscar innovación o valor agregado a las mismas. El manejo adecuado de los costos permite analizar el reconocimiento final del activo para determinar la ganancia o pérdida según los lineamientos de la NIIF.

\section{REFERENCIAS CONSULTADAS}

Arias, L., Portilla, L., \& Fernández, S. (2010). La distribución de costos indirectos de fabricación factor clave al costear un producto. [The distribution of indirect manufacturing costs is a key factor in costing a product]. Scientia Et Technica, 80. Obtenido de https://n9.cl/wns6

Arrelano, O., Quispe, G., Ayaviri, D., \& Escobar, F. (2017). Estudio de la Aplicación del Método de Costos ABC en las Mypes del Ecuador. [Study of the Application of the ABC Cost Method in the Mypes of Ecuador]. Revista de Investigaciones Altoandinas, 19(1), 33-46.

Bustamante, A. (2015). Costeo Basado en Actividades - ABC: Revision de Literatura. [Activity Based Costing - ABC: Literature Review. CEA]. CEA, 4. Obtenido de https://n9.cl/rxep

Caba, C., \& Caba, E. (2008). NIC 41 Agricultura. [IAS 41 Agriculture. International accounting standards]. Normas internacionales de contabilidad. Obtenido de https://n9.cl/gr9s0

Comité de Normas Internacionales de Contabilidad (IASCF). (Febrero de 2001). Normas Internacionales de Contabilidad 41 Agricultura [International Accounting Standards 41 Agriculture.]. Obtenido de https://n9.cl/gfdv

Cruz, A. (2006). Principales factores que afectan la prolificidad del ganado vacuno en latinoamerica. [Main factors that affect the prolificacy of cattle in Latin America. Put it on: REDVET, Electronic Veterinary Magazine]. Mágala: REDVET, Revista Electronica de Veterinaria. Obtenido de https://n9.cl/kr96 
Tania Mercedes Ortiz-Valencia; Verónica Paulina Moreno-Narváez; Jaime Fabián Díaz-Córdova

Del Río, C. (1996). Costos III : variable, A.B.C., sistema de costos, toma de decisiones, casos de costos. [Costs III: variable, A.B.C., cost system, decision making, cost cases]. México: Ecafsa.

Delicio, F. (1998). Costos Basados en la actividad [Activity-based costs]. Faces, 79. Obtenido de https://url2.cl/fgrQd

Erazo, J. C., \& Narváez, C. I. (2020). La gestión del capital intelectual y su impacto en la efectividad organizacional de la industria de cuero y calzado en la Provincia de Tungurahua - Ecuador. [The management of intellectual capital and its impact on the ] Recuperado de: https://n9.cl/52li. Revista Espacios, 254-271.

Galarza, M., Narváez , I., \& Erazo, J. (2019). Costeo ABC /ABM como herramienta de control en la gestión empresarial de la Organización de Pueblos de Chibuleo (ONOPUCH). [Abc Cost/ABM as a control tool in the business management of the Organization of Peoples of Chibuleo (ONOPUCH). Revista Arbitrada Interdisciplinaria Koinonía, 4(2), 114-146.

García, K., López, M., \& Ceron, H. (2015). Efecto de la NIC 41 en la actividad agrícola en Colombia. [Effect of IAS 41 on agricultural activity in Colombia. Colciencias]. Colciencias, 6. Obtenido de https://n9.cl/mffdp

Garzón, C. (2015). Determinación del costo de produccion y estados financieros para el sector ganadero bajo la normativa contable vigente en el Ecuador. Obtenido de [Determination of the cost of production and financial statements for the livestock sector under accounting regulations in force in Ecuador]: https://n9.cl/x4wc

Hernández, J., Núñez, I., \& Zapata, D. (2017). Criterios de medición y revelación de la NIC 41 aplicados por empresas perianas y chilenas. [Measurement and disclosure criteria of IAS 41 applied by Perian and Chilean companies]. Teuken Bidikay. Revista Latinoamericana de Investigacion en Organizaciones, ambiente $y$ sociedad, 3-4. Obtenido de https://n9.cl/bajp

Hernández, R., Fernández, C., \& Baptista, P. (2004). Metodología de la investigación. [Investigation methodology. Mexico.]. México. Obtenido de https://n9.cl/4tmw 
Tania Mercedes Ortiz-Valencia; Verónica Paulina Moreno-Narváez; Jaime Fabián Díaz-Córdova

Jara, J., \& Maldonado, H. (Agosto de 2011). Análisis y aplicación de un modelo de productividad para empresas del sector extractor de leche cruda caso: Agroindustrial "Las Lolas". [Analysis and application of a productivity model for companies in the raw milk extractor sector]. Obtenido de https://n9.cl/4mgdv

Jiménez, A. (2017). NIC 41: Ganado lechero. [Dairy cattle]. Obtenido de https://n9.cl/r4bx

KPMG Auditores Consultores Ltda. (2008). Activos biologicos y practicas. Europeas [Biological assets and European practices]. Obtenido de https://n9.cl/daci

Lalangui, M., Eras, R., \& Burgos, J. (2017). Costos de producción: estimación y proyección de ingresos. [Production costs: estimation and projection of income]. Machala: Utmach. Obtenido de https://n9.cl/tfol5

Mafla, A. (Diciembre de 2015). Sistema de contabilidad agropecuaria para la finca San Luis en el cantón Tulcán. [Agricultural accounting system for the San Luis farm in the Tulcán canton]. Obtenido de https://n9.cl/7awd

Mogrovejo , A., Narváez , C., \& Erazo , J. (2019). Factores determinantes del costeo ABC $A B M$ en la industria de textiles: Caso: Fábrica de textil Multijeans. [Determining factors of $A B C A B M$ costing in the textile industry: Case: Multijeans textile factory]. Revista Arbitrada Interdisciplinaria Koinonía, 4(2), 214-240. http://dx.doi.org/10.35381/r.k.v4i2.473

Morales, S., Morales, M., Andrade, A., \& Cevallos, L. (2020). Analítica de datos puros dentro del ámbito productivo y reproductivo de las ganaderías lecheras. [Analysis of pure data within the productive and reproductive field of milk herds]. Revista Arbitrada Interdisciplinaria Koinonía, 5(9), 287-310. http://dx.doi.org/10.35381/r.k.v5i9.649

Peralta , L., Juscamaita, J., \& Meza, V. (2016). Obtención y caracterización de abono orgánico líquido a través del tratamiento de excretas del ganado vacuno. [Obtaining and characterizing liquid organic fertilizer a through the treatment of excrets of beef cattle]. Ecología Aplicada, 15(1), 1-10.

Pozo, L., \& Sarmiento, L. (2012). Implementación de un sistema de contabilidad agropecuaria. [ Implementation of an agricultural accounting system]. Obtenido de https://n9.cl/h2lz7 
Tania Mercedes Ortiz-Valencia; Verónica Paulina Moreno-Narváez; Jaime Fabián Díaz-Córdova

Quizhpi, R., Nárvaez, C., \& Erazo, J. (2019). La gestión de las empresas comerciales en el marco de las NIFF para PYMES. [The accounting managment of commercial companies within the framework of IFRS for SMes]. Revista Arbitrada Interdisciplinaria Koinonía, 4(2), 265-298. http://dx.doi.org/10.35381/r.k.v4i2.475

Ramírez, D. (2008). Contabilidad administrativa. [Administrative accounting]. México: McGraw-Hill/Interamericana editores S.A de C.V. Obtenido de https://n9.cl/yk85

Reyes, P. E., Narváez, C. I., Erazo, J. C., \& Giler, L. V. (2020). Configuración del impuesto a la patente municipal con base al ingreso de las actividades económicas. Caso: GAD Municipal de Pucará - Ecuador. Revista Espacios, 197-211. 